\title{
Subpixel-shifted satellite images superresolution: software implementation
}

\author{
Sergey A. StankeVich ${ }^{1}$, Mikhail A. Popov ${ }^{1}$, Sergiy V. ShKLyar ${ }^{1}$, \\ KONSTANTIN Y. SUKHANOV ${ }^{1}$, ARTEM A. ANDREIEV ${ }^{1}$, ARTUR R. LYSENKO ${ }^{1}$, \\ XING KUn $^{2}$, CAO SHIXIANG ${ }^{2}$, SHI YUPAN ${ }^{2}$, ZHANG XING $^{2}$, Sun BOYA ${ }^{3}$ \\ ${ }^{1}$ Scientific Centre for Aerospace Research of the Earth \\ National Academy of Sciences of Ukraine \\ 55-B Oles Gonchar str., Kiev, 01054, UKRAINE \\ ${ }^{2}$ Beijing Institute of Space Mechanics and Electricity \\ 99 Zhongguancun East Road, Haidian District, Beijing, 100190, CHINA \\ ${ }^{3}$ CASICloud-Tech Co., Ltd. \\ 303 3F 51A Yongding Road, Haidian District, Beijing, 100048, CHINA
}

\begin{abstract}
The software implementation of superresolution of subpixel-shifted satellite images is described in this paper. This software implementation is carried out as two independent (standalone) software modules: first one - for preliminary co-registering and integer-pixel cropping of input satellite imageset, and second one - for superresolution itself of subpixel-shifted satellite images, that are the first module output. Software modules are based on the correlation algorithms, statistical regularization, and filtering in frequency domain. Both modules are designed using advanced computing solutions. The developed modules were tested on actual satellite images and were demonstrated the quite sufficient resolution enhancement.
\end{abstract}

Key-Words: satellite image, subpixel shift, superresolution, software module, frequency domain, modulation transfer function.

Received: July 14, 2019. Revised: December 31, 2019. Accepted: January 27, 2020. Published: March 5, 2020.

\section{Introduction}

Spatial resolution is the most important criterion of remote sensing capabilities. Regardless of the continuous improvement of remote sensing imaging systems, in many cases a lack of spatial resolution is evident. Spatial resolution enhancement makes remote sensing applications more successful, so such enhancement is important and relevant now. However, the possible resolution of the remote sensing imagers' hardware is limited by the microminiaturization technology of semiconductor sensor arrays on the one hand and the maximum allowable size of the lens on the other hand. Nevertheless, the superresolution can be engaged too by joint processing of several subpixel-shifted images of the same scene [1].

There are a significant number of superresolution methods known that use multiple low-resolution input images. These methods can be executed in both the spatial and frequency (Fourier or wavelet) domains. Both types, according to the models used, are subdivided into direct and probabilistic [2]. The first ones apply algebraic operations over input images, such as shift and add, while the last ones engage statistical techniques, such as maximum likelihood, Bayesian inference, random fields, etc.

Direct methods are not very effective for noisy images [3]. All other factors being equal, the Fourier domain processing usually consumes less computational burden [4]. That is why the fullframe statistical regularization in the frequency domain was chosen to perform superresolution in current research.

\section{General dataflow}

In a modern interpretation, the concept of spatial resolution is essentially statistical, as its value is estimated by statistical methods with multiple measuring in test digital aerospace images [5]. Practical experience evidences the direct connection between the spatial resolution of the imaging system and the probability of objects' detecting and recognizing in the acquired images [6].

In current research, the spatial resolution enhancement is reached by joint processing of imageset of the same scene, captured simultaneously. The main idea of this approach is extracting particular scene details from different images due to subpixel shift between them. All 
input images shifted relatively each other less than on one pixel are fused into joint superresolution image.

The technique for satellite imagery superresolution using a subpixel shift requires the transition from pixel grid of low spatial resolution images to a joint subpixel grid. Then the value in pixels of superresolution image can be estimated [7].

Subpixel shift between the low resolution images must be either known or estimated. Reference segments with high-contrast edges within the image are required for subpixel shift estimation. Under the linear affine transformation assumption, the shift value estimation can be performed using the discrete Fourier transform (DFT) in the frequency domain [8].

The general image processing dataflow for superresolution includes three stages: 1) acquiring several subpixel-shifted input images; 2) assigning a joint subpixel grid for all input images; 3 ) restoring the superresolution image from the input ones on the subpixel grid. The image processing sequence is described in more detail by the Fig. 1 flowchart.

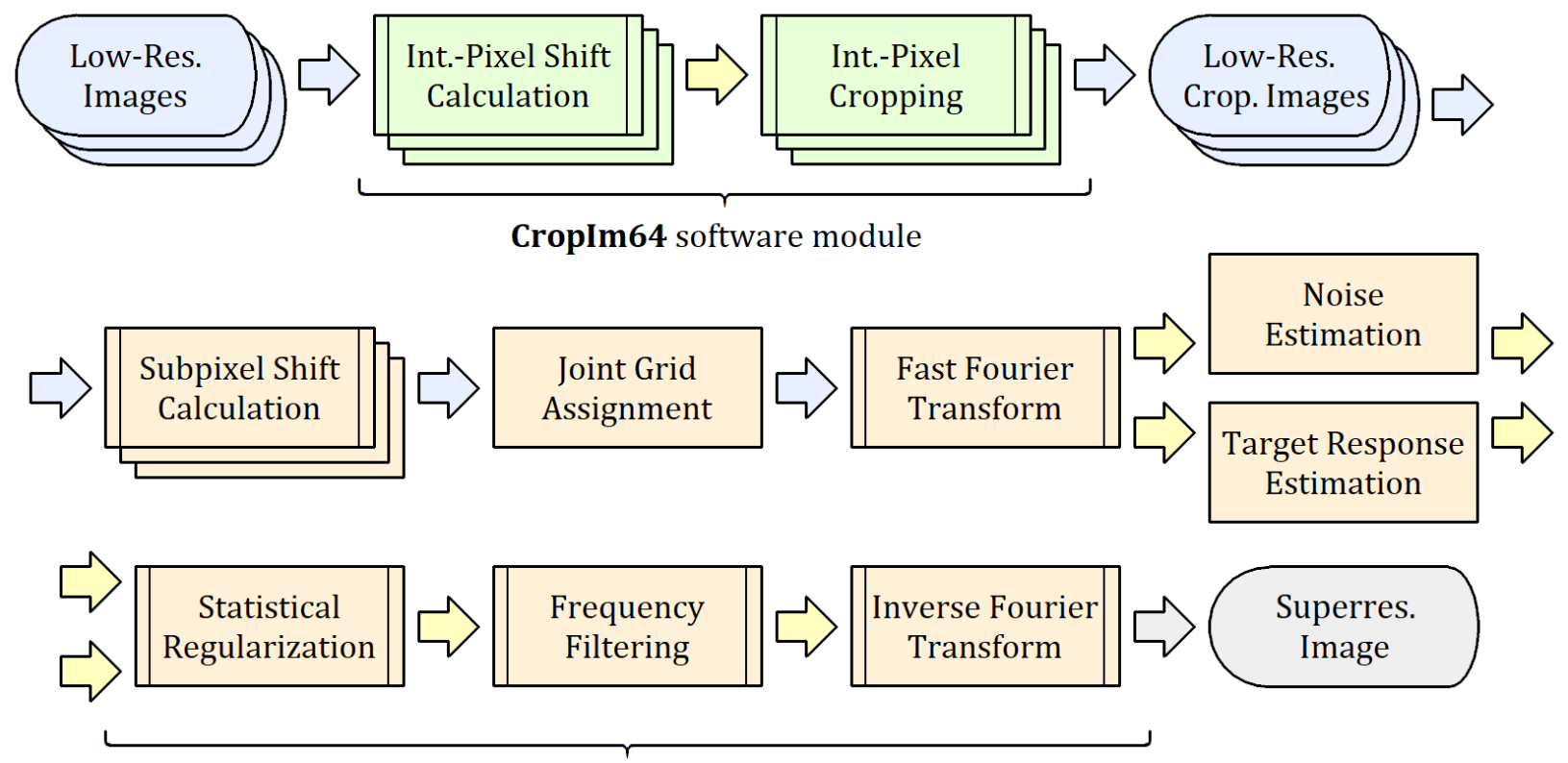

EnhIm64 software module

Fig. 1 Subpixel-shifted satellite images superresolution flowchart

The first stage is done by co-registering and cropping the input images. The correlation in frequency domain is used for integer-pixel shift estimation.

The joint subpixel grid of co-registered images is determined in the second stage so that as the minimum possible number of pixels of the input images overlap each subpixel of grid. Each coordinate subpixel shifts matrix for all input images should be estimated to do this [9].

After the subpixel shift of each of the input resampled images is determined, it becomes possible to re-interpolate them onto a joint subpixel grid. Then the superresolution image restoration in the third stage can be carried out [10]. Because all mutual subpixel shifts of the input images are already known, it is possible to project them onto a joint subpixel grid. Then, the resulting value is formed in each subpixel from the set of values corresponding to the input images. In this way, the output enhanced resolution image is obtained.

As for the software implementation, the input image integer-pixel co-registering and cropping operations are performed by a standalone software module (CropIm64 in Fig 1), but the resolution enhancement itself - by another standalone software module (EnhIm64 in Fig 1).

\section{Software architecture}

The proposed method provides the eligible level of resolution enhancement only if input images meet specific requirements, such as the consistency of scale over the field of whole image [11], the prohibition of angular misalignment of input images, and the area of overlap of the input images is at least $90 \%$ [12].

Also, it should be mentioned, that using images with a large area of cloud cover, as well as images 
obtained with a long-time interval, could decrease images' processing performance [13].

\subsection{Correlation-based algorithm for determining the subpixel shift between images}

The mutual integer-pixel shift between two images of the same scene implies that the second image is considered as a parallel transfer from the first one.

Determining the integer-pixel shift between images is the task of finding the true shift value, which corresponds to the maximum correlation between the input images [14].

The input data are two $Y_{1}$ and $Y_{2}$ images of $m \times n$ dimension at $(x, y)$, points, $x=0,1, \ldots, m-1, y=0,1$, $\ldots, n-1$. The pixel size is selected as a linear unit.

Discrete Fourier transform is computed by the following equation:

$\hat{Y}_{k}(\theta, \vartheta)=\sum_{x=0}^{m-1} \sum_{y=0}^{n-1} Y_{k}(x, y) e^{-2 \pi i\left(\theta x+y_{y}\right)}$,

where $k=1$ or $k=2$ under $\theta=\ldots,-\frac{2}{m},-\frac{1}{m}, 0$, $\frac{1}{m}, \frac{2}{m}, \ldots$ and $\vartheta=\ldots,-\frac{2}{n},-\frac{1}{n}, 0, \frac{1}{n}, \frac{2}{n}, \ldots$

$\hat{Y}_{k}(\theta, \vartheta)$ values will be considered for $|\theta|<\frac{1}{2},|\vartheta|<\frac{1}{2}$ only.

To calculate the correlation between the images, the Parseval equation is used: if $\hat{Y}_{k}(\theta, \vartheta)$ is the Fourier transform of the $Y_{k}(x, y)$, function defined for $\quad 0 \leq x \leq m, 0 \leq y \leq n, \quad$ that is $\hat{Y}_{k}(\theta, \vartheta)=\int_{0}^{m} \int_{0}^{n} Y_{k}(x, y) e^{-2 \pi i\left(\theta_{x}+\vartheta_{y}\right)} d x d y$, then

$\int_{0}^{m} \int_{0}^{n} Y_{1}(x, y) Y_{2}(x, y) d x d y=$

$=\sum_{\theta=\ldots, \frac{1}{m}, 0, \frac{1}{m}, \ldots, \theta_{1}=\ldots, \frac{1}{n}, 0, \frac{1}{n}, \ldots .} \hat{Y}_{1}(\theta, \vartheta) \hat{Y}_{2}(\theta, \vartheta)$.

The Fourier transform of the second image shifted by $(\Delta x, \Delta y)$, after suppressing the highfrequency component will be

$$
\hat{\boldsymbol{F}}_{2}^{(\Delta x, \Delta y)}(\theta, \vartheta)=\hat{\boldsymbol{F}}_{2}(\theta, \vartheta) e^{2 \pi i(\theta \Delta x+\vartheta \Delta y)} \text {. }
$$

The objective function is defined as

$$
\begin{aligned}
& Q(\Delta x, \Delta y)=\sum_{\theta=\ldots, \frac{1}{m}, 0, \frac{1}{m}, \ldots, \theta_{1}=\ldots,-\frac{1}{n}, 0, \frac{1}{n}, \ldots} \hat{F}_{1}(\theta, \vartheta) \hat{F}_{2}^{(\Delta x, \Delta y)}(\theta, \vartheta)= \\
& =\sum_{k=-\lfloor m / 2\rfloor l=-\lfloor n / 2\rfloor}^{\lfloor m / 2\rfloor} \sum_{1}^{\lfloor n / 2\rfloor} \hat{F}_{1}\left(\frac{k}{m}, \frac{l}{n}\right) \hat{F}_{2}\left(\frac{k}{m}, \frac{l}{n}\right) \exp \left[2 \pi i\left(\frac{k \Delta x}{m}+\frac{l \Delta y}{n}\right)\right] .
\end{aligned}
$$

The equation solving toward to $(\Delta x, \Delta y)$ with integrity constraint provides the desired integerpixel shift value between the images.

\subsection{Co-registering and cropping of integer- pixel shifted images}

Co-registration of integer-pixel shifted images is performed in three stages: 1) searching for image shifts and compilation of shift matrices along the $X$ and $Y$ axes; 2) assign a reference image, in which the area of overlap with other images will be the largest referring to image size; and 3) cropping all input images.

At the first stage, the described algorithm is applied to all input images pairwise. That is, this procedure is performed $n-1$ times with the number of images $n$. Thus, the shift values are calculated for each of the axes. Then, two matrices, the elements of which are shifts along the $X$ and $Y$ axes, respectively, are formed. As an example, only the shift matrix along the $X$ axis will be considered. Let $D_{X}$ be the shift matrix along the $X$ axis

$D_{X}=\left(\begin{array}{c}\Delta x_{11} \cdots \Delta x_{1 n} \\ \cdots \\ \Delta x_{n 1} \cdots \Delta x_{n n}\end{array}\right)$,

where $\Delta x_{i j}$ is the shift of image $i$ referring to image $j$ along the $X$ axis.

At the second stage, the searching for a reference image, the shift of the remaining images relative to which is the smallest, takes place. This is necessary so that when co-registering images, the smallest part of the image is cropped, in turn, it allows to select the largest overlap area. To determine such a reference image, the sum of the minimum and maximum shift for each row of the $D_{X}$ matrix is calculated. The smallest sum will correspond to the desired reference image.

At the last stage, images are cropped and coregistered. After selecting the reference image, the search for indices of new images is performed, taking into account the row of the shift matrix, which corresponds to the selected reference image:

$X_{i}^{\prime}=X_{i}\left[\max \left(\Delta x_{\mathrm{ref}}\right)-\Delta x_{\text {ref } i} ; \Delta x_{\mathrm{ref} i}-\max \left(\Delta x_{\mathrm{ref}}\right)\right]$, where $X_{i}^{\prime}$ is cropped $i$ image, $X_{i}$ is initial $i$ image, ref is a selected reference image, $\Delta x_{\text {ref } i}$ is the shift along the $X$ axis of $i$ image referred to the reference image $\left(\Delta x_{\text {ref } i}\right.$ corresponds to element (ref, $i$ ) of matrix $D_{X}$ ), $\Delta x_{\text {ref }}$ is a set that contains the shift values of all images referred to the reference image $\left(\Delta x_{\text {ref }}\right.$ corresponds to row ref of matrix $\mathrm{D} x),\left[\max \left(\Delta x_{\mathrm{ref}}\right)-\right.$ $\left.\Delta x_{\text {ref } i} ; \Delta x_{\text {ref } i}-\max \left(\Delta x_{\text {ref }}\right)\right]$ are the coordinates of the input image that correspond to the cropped image. 
For cropping along the $Y$ axis, the same operations to those described above, are performed.

\subsection{Superresolution algorithm}

The superresolution image is acquired from several sub-pixel shifted low-resolution images in next steps: preparing sub-pixel shifted low-resolution images, finding subpixel-shift value for each pair of low-resolution images, as in 3.2 subsection, estimating the autocovariance matrix of lowresolution images, estimating the autocovariance matrix of the noise image [15], determining the transfer functions [16] and creating super-resolution image [17].

The autocovariance matrix of low-resolution images can be a mean sum of the Fourier transforms of each source image autocovariance matrix [18].

The noise image can be retrieved by projecting each source low-resolution image onto common superresolution grid and evaluating the noise as the mean value of the sum of each pair-wise projected low-resolution images difference.

Assume we have $N$ source images. Let $U$ and $V$ be matrices that correspond to shifts between source images, where $u_{i j}$ and $v_{i j}$ are horizontal and vertical shift between $i$-th and $j$-th source images respectively. The mean shift matrix $M_{i j}$ between all images is:

$M_{i 1}=\sum_{j} u_{i j}, M_{i 2}=\sum_{j} v_{i j}, i=\overline{1, N}, j=\overline{1, N}$.

The aligned shift matrix $A$, which is projected onto joint grid, can be found as:

$A_{1 j}^{\sin }=\sum_{i} \sin \left[(4 \pi M)_{i j}\right] A_{1 j}^{\cos }=\sum_{i} \cos \left[(4 \pi M)_{i j}\right]$,

$i=\overline{1, N}, j=\overline{1,2}$;

$A_{1 j}=\frac{\operatorname{atan}\left(A_{1 j}^{\sin }, A_{1 j}^{\cos }\right)}{4 \pi}, j=\overline{1,2}$.

The optimized shift matrix $O$ will be:

$O_{i j}=M_{i j}-A_{1 j}, i=\overline{1, N}, j=\overline{1,2}$.

Let $I$ be the $m \times n$ source image. Thus, shifted image $S$ becomes optimized along vertical and horizontal axis by following approach.

For vertical shift:

$$
S_{i}^{k}=\left\{\begin{array}{c}
\left(1-O_{k}\right) I_{i j}+O_{k} I_{i+1, j}, O_{k}>0, \\
i=\overline{1, m-1}, j=\overline{1, n}, k=\overline{1, N} \\
-O_{k} I_{i-1, j}+\left(1+O_{k}\right) I_{i j}, O_{k} \leq 0, \\
i=\overline{2, m}, j=\overline{1, n}, k=\overline{1, N}
\end{array} .\right.
$$

For horizontal shift:

$$
S_{i}^{k}=\left\{\begin{array}{l}
\left(1-O_{k}\right) I_{i j}+O_{k} I_{i, j+1}, O_{k 2}>0, \\
i=\overline{1, m}, j=\overline{1, n-1}, k=\overline{1, N} \\
-O_{k} I_{i, j-1}+\left(1+O_{k}\right) I_{i j}, O_{k 2} \leq 0, \\
i=\overline{1, m}, j=\overline{2, n}, k=\overline{1, N}
\end{array},\right.
$$

where $k$ is the source image number, $O_{k}$ is the vertical or horizontal shift.

The noise image is a mean sum of all pair-wise differences between $S^{k}, k=\overline{1, N}$.

Now, let $X$ and $Y$ be the enhanced superresolution and low-resolution image matrices accordingly. In order to restore enhanced resolution image the transfer function, which describes the enhancing process, is needed. When restoring the high-resolution pixel from several shifted lowresolution pixels the model looks as described below:

$Y(y, x)=G(\Delta y, \Delta x) \otimes X(y, x)$,

$-0.5 \leq \Delta y \leq 0.5,-0.5 \leq \Delta x \leq 0.5$,

$G(\Delta y, \Delta x)=\left(\begin{array}{ccc}(0.5-\Delta y)(0.5-\Delta x) & (0.5-\Delta y) & (0.5-\Delta y)(0.5+\Delta x) \\ (0.5-\Delta x) & 1 & (0.5+\Delta x) \\ (0.5+\Delta y)(0.5-\Delta x) & (0.5+\Delta y) & (0.5+\Delta y)(0.5+\Delta x)\end{array}\right)$

- is the general convolution matrix of superresolution transform.

The frequency domain transfer function is:

$T(\eta, \xi)=\left((0.5-\Delta y) e^{-2 \pi i \eta / m}+1+(0.5-\Delta y) e^{2 \pi i \eta / m}\right) \times$

$\times\left((0.5-\Delta x) e^{-2 \pi i \xi / n}+1+(0.5-\Delta x) e^{2 \pi i \xi / n}\right)$.

The super-resolution image model is:

$4 \widehat{Y}_{k}=T_{k}(\eta, \xi) \hat{X}(\eta, \xi)+T_{k}(\eta \pm m, \xi) \hat{X}(\eta \pm m, \xi)+$

$+T_{k}(\eta, \xi \pm n) \hat{X}(\eta, \xi \pm n)+T_{k}(\eta \pm m, \xi \pm n) \times$

$\times \hat{X}(\eta \pm m, \xi \pm n)+4 \hat{E}(\eta, \xi)$,

where $\hat{Y}_{k}$ is the Fourier transform of the $k$-th lowresolution image, $\hat{X}(\eta, \xi)$ is the Fourier transform of the superresolution image, $\widehat{E}_{k}(\eta, \xi)$ is the Fourier transform of the error in the $k$-th observed image.

\section{Developed software modules}

Developed software operates on 64-bit graphic workstation and consists of two independent (standalone) software modules: a module for coregistration of satellite images and a module for superresolution of subpixel-shifted satellite images. Software modules are developed in the $\mathrm{C}++/ \mathrm{CLI}$ programming language using TCL (GUI), OpenCV (graphic), fftw (fast Fourier transform) and GDAL (geospatial) open libraries. Both modules transact image processing in the frequency domain and has a 
graphical user interface (GUI) which is shown in the Fig. 2.

It is possible to combine the functionality of both modules into a single one, however, in our opinion, this will slightly limit the image processing flexibility, as the module for automatic cropping of satellite images has an inherent value. Two coupled modules in conjunction form a complete pipeline of satellite image processing

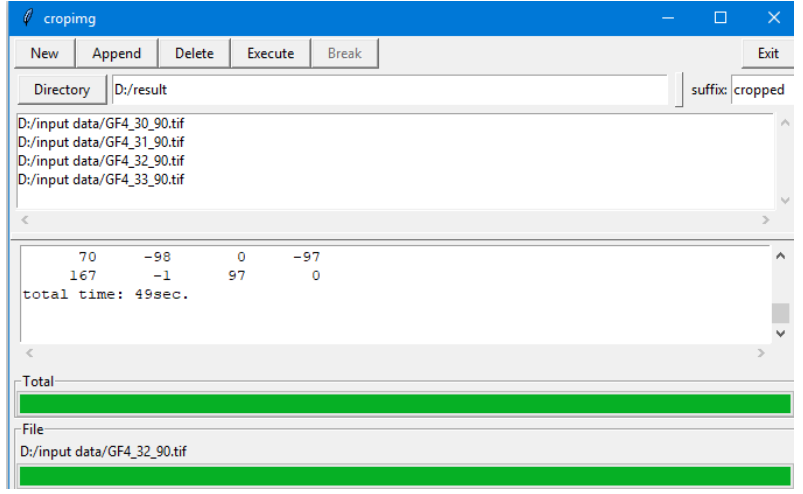

$a$

\begin{tabular}{|c|c|}
\hline Image enhancement & $\square$ \\
\hline $\begin{array}{l}\text { E:IVSProjectsImageEnhancementFilesIGF4_30_cropped.tif } \\
\text { E:IVSProjectsVmageEnhancementFilesIGF4_31_cropped.tif } \\
\text { E:IVSProjectsVmageEnhancementFilesIGF4_32_cropped.tif } \\
\text { E:IVSProjectsVmageEnhancementFilesIGF4_33_cropped.tif }\end{array}$ & Open images \\
\hline E:IVSProjectsVImageEnhancementFilesIGF4_result.tif & Save image \\
\hline Stop & $18 \%$ \\
\hline Evaluating image shift... & \\
\hline Show options & \\
\hline
\end{tabular}

Fig. 2 Developed software GUI:

$a$ - module for integer-pixel co-registration of satellite images, $b$ - module for subpixel-shifted satellite images superresolution

The output of the first software module is integer-pixel co-registered and uniformly cropped input imageset. The second software module produces a single superresolution satellite image from input integer-pixel cropped imageset.

The developed software module uses satellite images of arbitrary pixel size, shifted by an integerpixel value (first step) and by subpixel value (next step), as input data. The source data can be stored in the following file formats: single-band uncompressed TIFF / GeoTIFF of 8 bits per pixel (byte), 16 bits per pixel (uint) and 32 bits per pixel (float) types; image files' permissible extensions are .tif, or .tiff

\section{Developed software testing}

For the experiment, four test fragments of same scene Gaofen-4 single-band satellite images (Fig. 3) were used:

- GF4_B1_E116.5_N39.9_20191001_L1A0000260530, $4551 \times 3622$ pixels size;

- GF4_B1_E116.5_N39.9_20191001_L1A0000260531, $4201 \times 33792$ pixels size;

- GF4_B1_E116.5_N39.9_20191001_L1A0000260532, $4551 \times 33692$ pixels size;

- GF4_B1_E116.5_N39.9_20191001_L1A0000260533, $4551 \times 33502$ pixels size.
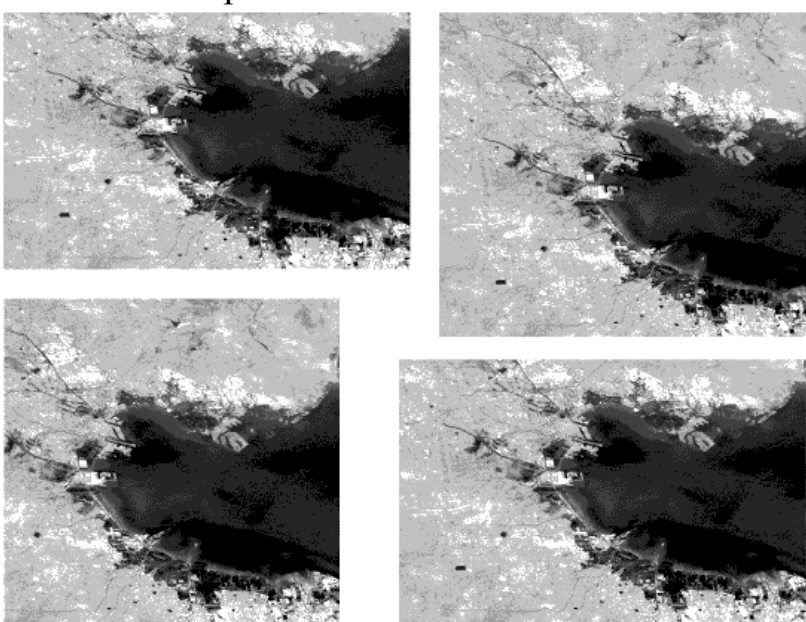

Fig. 3 Test fragments of Gaofen-4 single-band satellite images

The output size of each of the co-registered and cropped images is $4190 \times 3335$ pixels. One of the integer-pixel cropped image is shown in the Fig. 4

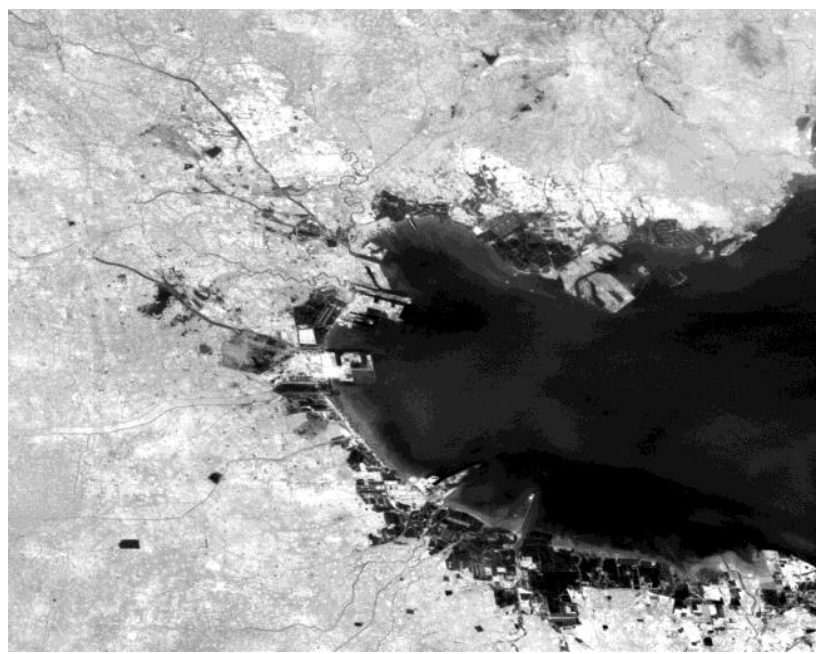

Fig. 4 Integer-pixel cropped test satellite image

The enhanced resolution image obtained by superresolution module from the cropped imageset is shown in the Fig. 5. The output image has much more details than the input ones. Picture Fig. 5 contains sequentially enlarged satellite image 
fragments: input ones are on the left, and superresolution results are on the right.

\section{Results evaluating}

The result demonstrated in previous section where evaluated objectively [19]. The achieved actual resolution of images was assessed through the predetermined threshold of modulation transfer function (MTF) [20]. The MTF was determined as a Fourier transform of the point spread function (PSF) of image. The PSF, in turn, was measured by an edge spread function (ESF) extracted directly from the image [21].
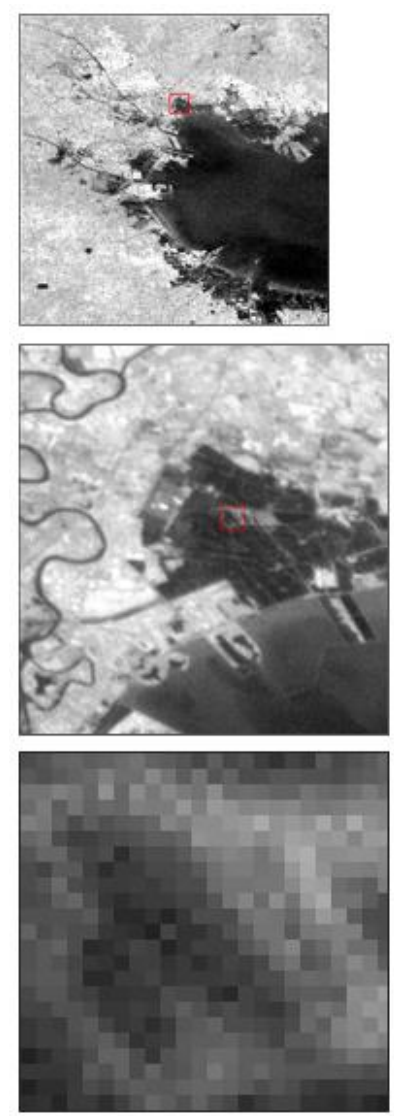

$a$
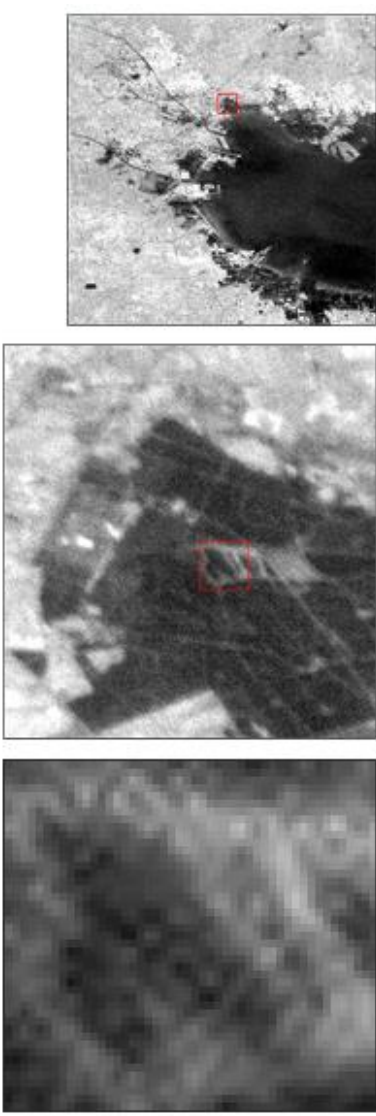

b
Fig. 5 Satellite image superresolution demo: $a$-input image fragment, $b$-superresolution image fragment

Our approach involves statistical filtering of a large number of ECF realizations captured by window scanning of the image, as well as the optimal Gaussian approximation of the ESF, taking into account the radiometric values of its points [22]. Resolutions are determined for horizontal and vertical ESFs separately. Plot Fig. 6 displays averaged normalized ESFs, automatically extracted from actual satellite image.

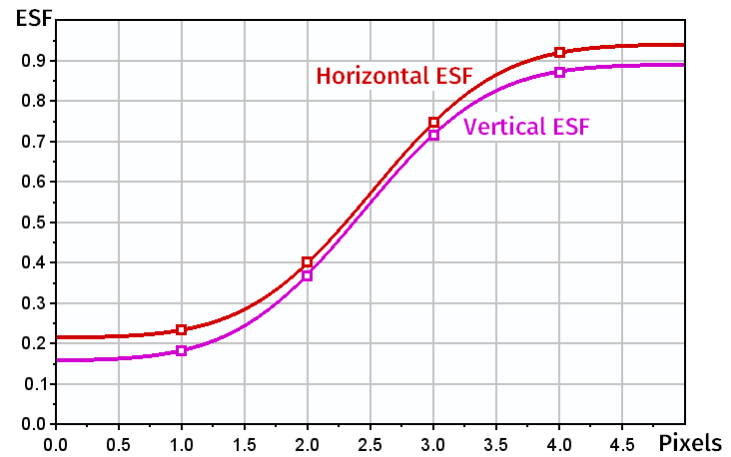

Fig. 6 Normalized ESFs of satellite image

For input test images, the averaged resolutions are: horizontal -3.95 pixels, vertical -3.78 pixels, isotropic -3.86 pixels. The same values for the output superresolution image are 1.92 pixels, 2.05 pixels and 1.98 pixels, respectively. Thus, the actual resolution of the test image after processing improved by $48.7 \%$.

\section{Conclusion}

Summing up, this paper describes the theoretical foundations, algorithms and developed software modules for subpixel-shifted satellite images superresolution. The software implementation of superresolution is embodied as two independent modules: a module for satellite images coregistration and a module for subpixel-shifted satellite images superresolution. Both modules transact image processing within the frequency domain. Each module is a GUI-based standalone 64bit executable.

The developed software was tested with actual satellite images and a $48.7 \%$ improvement in objective spatial resolution was demonstrated, which is a quite reasonable result.

Future works should be focused on improving superresolution algorithms in the frequency domain, as well as on optimizing the program code of modules to enhance software performance, especially in the huge-size satellite images processing.

\section{References:}

[1] V.I. Lyalko, M.A. Popov, S.A. Stankevich, S.V. Shklyar, V.N. Podorvan, N.I. Likholit, V.M. Tyagur, C.V. Dobrovolska. Subpixel processing of images from the frame infrared camera for future Ukrainian remote sensing satellite system. Proceedings of the $10^{\text {th }}$ International Conference on Digital Technologies (DT'2014). Žilina, IEEE, 2014, pp. 232-235. DOI: 10.1109/DT.2014.6868717

[2] L. Morera-Delfín, R. Pinto-Elías, H.J. OchoaDomínguez. Overview of super-resolution techniques. In: O. Vergara Villegas, M. Nandayapa, 
I. Soto (Eds). Advanced Topics on Computer Vision, Control and Robotics in Mechatronics. Springer Nature, 2018, pp. 101-127. DOI: 10.1007/978-3319-77770-2_5

[3] M.M. Khattab, A.M. Zeki, A.A. Alwan, A.S. Badawy. Regularization-based multi-frame superresolution: A systematic review. Journal of King Saud University - Computer and Information Sciences, 2020, vol. 32, no. 7, pp. 755-762. DOI: 10.1016/j.jksuci.2018.11.010

[4] P. Vandewalle, S. Süsstrunk, M. Vetterli. A frequency domain approach to registration of aliased images with application to super-resolution. EURASIP Journal on Advances in Signal Processing, 2006, vol. 2006, ID. 071459, 14 p. DOI: 10.1155/ASP/2006/71459

[5] A. Descloux, K.S. Grußmayer, A. Radenovic. Parameter-free image resolution estimation based on decorrelation analysis. Nature Methods, 2019, vol. 16, no. 9, pp. 918-924. DOI: 10.1038/s41592-019$0515-7$

[6] V.I. Kononov. Relation of information and probabilistic estimates of imaging systems (in Russian). Optical and Mechanical Industry, 1991, vol. 58, no. 11, pp. 13-18.

[7] S.A. Stankevich, S.V. Shklyar, V.N. Podorvan, M.S. Lubskyi. Thermal infrared imagery informativity enhancement using sub-pixel co-registration. Proceedings of the International Conference on Information and Digital Technologies (IDT 2016). Rzeszów, IEEE, 2016, pp. 245-248. DOI: 10.1109/DT.2016. 7557181

[8] H.S. Stone, M.T. Orchard, E.-C. Chang, S.A. Martucci. A fast direct Fourier-based algorithm for subpixel registration of images. IEEE Transactions on Geoscience and Remote Sensing, 2001, vol. 39, no.10, pp. 2235-2243. DOI: 10.1109/36.957286

[9] S.A. Stankevich, A.A. Andreiev, A.R. Lysenko. Multiframe remote sensed imagery superresolution. Proceedings of the $15^{\text {th }}$ International ScientificPractical Conference on Mathematical Modeling and Simulation Systems (MODS 2020). Chernihiv, CNUT, 2020, pp. 49-52.

[10] M.A. Popov, S.A. Stankevich, S.V. Shkliar An algorithm for resolution enhancement of subpixel displaced images (in Russian). Mathematical Machines and Systems, 2015, no. 1, pp. 29-36.

[11] E.G. Voronin. On the displacements of the contours of the optic-electronic space images. Causes and evaluation of offsets (in Russian). Geodesy and cartography, 2017, vol. 923, no. 5, pp. 34-41. DOI: 10.22389/0016-7126-2017-923-5-34-41

[12] P. d'Angelo. Automatic orientation of large multitemporal satellite image blocks. Proceedings of International Symposium on Satellite Mapping Technology and Application (ISSMTA 2013). Nanjing, ISPRS, 2013, pp. 1-7.

[13] V. Ferraris, N. Dobigeon, Q. Wei, M. Chabert. Detecting changes between optical images of different spatial and spectral resolutions: A fusion- based approach. IEEE Transactions on Geoscience and Remote Sensing, 2018, vol. 56, no. 3, pp. 15661578. DOI: 10.1109/TGRS. 2017.2765348

[14] S.A. Stankevich, M.A. Popov, S.V. Shklyar, K.Y. Sukhanov, A.A. Andreiev, A.R. Lysenko, X. Kun, S. Cao, S. Yupan, S. Boya. Estimation of mutual subpixel shift between satellite images: software implementation. Ukrainian journal of remote sensing, 2020, no. 24, pp. 9-14. DOI: 10.36023/ujrs.2020.24.165

[15] S.V. Vaseghi. Advanced Digital Signal Processing and Noise Reduction. Chichester, John Wiley, 2006, 480 p. ISBN: 9780470094945

[16] G.D. Boreman. Modulation Transfer Functions in Optical and Electro-Optical Systems. Bellingham, SPIE Press, 2001, 120 p. ISBN: 9780819441430

[17] R.G. Driggers, E.L. Jacobs, S.S. Young. Signal Processing and Performance Analysis for Imaging Systems. Norwood, Artech House, 2008, 270 p. ISBN: 9781596932876

[18] A.C. Kak, M. Slaney. Principles of Computerized Tomographic Imaging. Philadelphia, SIAM, 2001, 340 p. ISBN: 9780898714944

[19] J. Dutta, S. Ahn, Q. Li. Quantitative statistical methods for image quality assessment. Theranostics, 2013, vol.3, no.10, pp.741-756. DOI: $10.7150 /$ thno. 6815

[20] D.P. Haefner. Best practices for imaging system MTF measurement. In: Proceedings of XVI IS\&T International Symposium on Electronic Imaging, Image Quality and System Performance (EI 2019). Burlingame: Society for Imaging Science and Technology, 2019, pp. 319-324. DOI: 10.2352/ISSN.2470-1173.2019. 10.IQSP-319

[21] C.D. Claxton, R.C. Staunton. Measurement of the point-spread function of a noisy imaging system. Journal of the Optical Society of America, 2008, vol.25, no.1, pp. 159-170. DOI: 10.1364/JOSAA.25.000159

[22] S.A. Stankevich. Evaluation of the spatial resolution of digital aerospace image by the bidirectional point spread function parameterization. In: S. Shkarlet, A. Morozov, A. Palagin (Eds). Advances in Intelligent Systems and Computing, vol.1265. Cham: Springer Nature, 2021, pp. 317-327. DOI: 10.1007/978-3030-58124-4_31

\section{Creative Commons Attribution License 4.0 (Attribution 4.0 International , CC BY 4.0)}

This article is published under the terms of the Creative Commons Attribution License 4.0 https://creativecommons.org/licenses/by/4.0/deed.en US 